\section{EDUCATION}

Research, Innovation and Solutions on-line
Electronic Journal of Research

in Educational Psychology

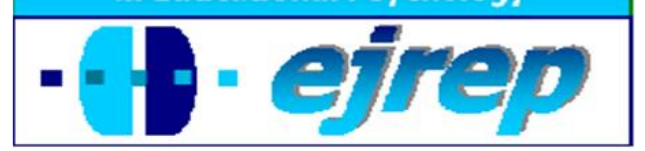

Editorial EOS

\title{
Una nueva mirada a la formación en eficacia docente de Gordon (TET): Un estudio- intervención en el aprendizaje social y emocional del profesorado
}

\section{Markus Talvio ${ }^{1}$, Kirsti Lonka ${ }^{1}$, Erkki Komulainen ${ }^{1}$, Marjo Kuusela $^{2}$ y Taru Lintunen ${ }^{2}$}

${ }^{1}$ Department of Teacher Education, University of Helsinki, Helsinki

${ }^{2}$ Department of Sport Sciences, University of Jyvaskyla, Jyvaskyla

\section{Finlandia}

Correspondencia: Markus Talvio, C/O Professor Kirsti Lonka, P.O. Box 9, 00014 University of Helsinki. Finland. E-mail: markus@markustalvio.com

C Education \& Psychology I+D+i and Editorial EOS (Spain) 


\section{Resumen}

Introducción.. En este estudio exploramos el desarrollo de las habilidades de aprendizaje social y emocional (SEL) de los docentes, mediante el uso de MET [Maestros Eficaz y Técnicamente preparados] (Gordon Training International) como intervención con dos grupos de docentes. También quisimos abordar el modelo de Gordon desde la perspectiva de la psicología educativa moderna. Los efectos de la intervención MET sobre los docentes fueron examinados utilizando el modelo de Kirkpatrick y Kirkpatrick (2006), ya que consideramos importante observar diversos aspectos de los resultados de la intervención, incluyendo las reacciones, el conocimiento, la aplicación del conocimiento (habilidades) y el bienestar general de los participantes.

Método. Los docentes participantes en el MET eran tutores $(n=20)$ de un centro de primaria y profesores de materia $(n=23)$ de un centro de secundaria de Finlandia. El grupo de comparación comprendió profesores de materia $(n=26)$ de un centro de secundaria que no participaron en MET. Las diferencias estadísticas de los resultados posteriores a la prueba se examinaron con ANOVA unidireccional de muestra dependiente.

Resultados. En el grupo de comparación no se encontraron diferencias entre las mediciones previas y posteriores a la prueba. Entre los participantes las reacciones hacia el MET fueron positivas. Además hubo resultados significactivos en otros dos aspectos: mejoraron tanto el conocimiento como la aplicación del conocimiento. El bienestar general de los docentes medido al final de la intervención mostró cambios menores.

Conclusión. MET pareció alcanzar sus objetivos, pues los docentes aprendieron a aplicar habilidades de SEL durante la intervención.

Palabras clave: aprendizaje social y emocional (SEL); formación de docentes; Maestros Eficaz y Técnicamente preparados (MET); método de afrontar la interacción desafiante (DCI); habilidades de interacción social; bienestar. 


\title{
Revisiting Gordon's Teacher Effectiveness Training: An Intervention Study on Teachers' Social and Emotional Learning
}

\begin{abstract}
Introduction. This study explored the development of teachers' social and emotional learning (SEL) skills by using Teacher Effectiveness Training (TET) (Gordon Training International) as an intervention with two groups of teachers. Further, Gordon's model was approached from the perspective of modern educational psychology. The effects of TET intervention on teachers were examined by utilizing Kirkpatrick and Kirkpatrick's model (2006), since we considered it important to look at various aspects of the outcomes of the intervention, including the participants' reactions, knowledge, knowledge application (skills) and overall well-being.
\end{abstract}

Method. The teachers participating in TET were class teachers $(n=20)$ from one elementary school and subject-matter teachers $(n=23)$ from one secondary school in Finland. The comparison group comprised subject-matter teachers $(n=26)$ from one secondary school who did not participate in TET. The statistical differences of the post-test scores were examined with dependent sample one-way ANOVA.

Results. In the comparison group, no differences between the pre- and post-test measurements were found. Among participants, reactions towards TET were positive. Further, there were significant results in two other aspects: both knowledge and knowledge application (skills) improved. The overall well-being of the teachers, measured at the end of the intervention, showed minor changes.

Conclusion. TET appeared to achieve its goals, since teachers learned to apply SEL skills during the intervention.

Keywords: social and emotional learning (SEL); teacher training; Teacher Effectiveness Training (TET); The Dealing with Challenging Interaction (DCI) method; social interaction skills; well-being 


\section{Introducción}

Es escasa la investigación acerca de cómo los docentes estudian, desarrollan y mejoran sus habilidades de interacción social, si bien es cierto que en la moderna psicología del aprendizaje se reconoce esas habilidades como herramientas claves en un entorno de aprendizaje. Las teorías socioconstructivistas de la Psicología Educativa enfatizan el papel activo del aprendiz (Lonka y Ahola, 1995), y, por lo tanto, es crucial que los docentes tengan las herramientas de apoyo para esa posición agéntica (Bandura, 2006; Edwards, 2005). La autonomía, la participación y la auto-eficacia son fundamentales en este marco (Bruner, 1996; Sfard, 1998; Vygotsky, 1978). Actualmente se cree que los alumnos deben experimentar la seguridad y auto-eficacia en interacciones con los miembros de la comunidad escolar (Pietarinen, Soini, y Pyhältö, 2010). Los educadores deben fijar retos que van más allá de las competencias existentes de los alumnos, y ajustar su nivel de apoyo según los alumnos rebasan las diversas fases de adquisición de habilidades, retirando gradualmente la ayuda según ellos se vuelven más diestros en el dominio de las tareas por sí mismos (p.ej., Bandura, 2006; Vygotsky, 1978).

En la teoría de la autodeterminación (SDT en inglés, TAD en castellano) (Ryan y Deci, 2000; Deci y Ryan, 2002), es importante también promover la autonomía, la creatividad y la motivación intrínseca. Desde esta perspectiva, una de las tareas principales del docente es la de respaldar la autonomía en sus alumnos (Leroy, Bressoux, Sarrazin, y Trouilloud, 2007). La TAD y las teorías relacionadas se centran cada vez más en la cognición constructiva y la inteligencia social que siempre ofrecen medios alternativos y oportunidades para abordar los problemas desde perspectivas nuevas y más fructíferas (Cantor, 2003). Leroy et al. (2007) sugirió que al usar las habilidades de interacción social se puede mejorar la motivación intrínseca de los alumnos. Ellos mostraron que cuando los docentes creían que se podía mejorar el logro académico de los alumnos a través de los propios esfuerzos de los alumnos, esto favorecía indirectamente el clima de apoyo a la autonomía. Era importante que los mismos docentes experimentaran la auto-eficacia y creyeran que podían fomentar el aprendizaje de sus alumnos.

Las teorías descritas anteriormente son muy cercanas al movimiento de psicología positiva que está basado en la psicología humanista y enfatiza las fortalezas y potenciales de los seres humanos, en lugar de sus debilidades y problemas (Aspinwall y Staudinger, 2003). 
Ya para los años sesenta, Thomas Gordon desarrolló un modelo para formar docentes en las habilidades de interacción social y basó su trabajo en la psicología humanista. Gordon (2003, págs. 107-108) declaró que "dentro de cada uno de nosotros hay un fuerte instinto hacia la independencia y la auto responsabilidad”, que enfatizan de forma implícita las capacidades de agencia. En su modelo, era fundamental la habilidad de escuchar, donde están presentes la aceptación de y el respeto por el alumno. Gordon sugirió que la escucha activa por parte del docente es una herramienta para promover el crecimiento de los alumnos hacia la autonomía, la responsabilidad propia, la autodeterminación, el autocontrol y la autoevaluación (Gordon, 2003, pág. 9). Los docentes deben, según él, alimentar y promover esas habilidades ya que no se desarrollan automáticamente.

En general, todas las teorías mencionadas anteriormente han impulsado en tiempos recientes la investigación sobre las habilidades de interacción social. También vemos una gran similitud con los valores implícitos del modelo de Gordon. Por ejemplo, Bird y Sultmann (2010) ven las habilidades de interacción social como herramientas para desarrollar relaciones, fomentar el bienestar y promover el aprendizaje de calidad. Ven que el ámbito educativo "tiene el potencial para atraer a los jóvenes en formas que les ayudarán a definir quiénes son, por qué son importantes, cómo deben actuar y quiénes pueden llegar a ser” (Bird y Sultmann, 2010, pág. 143). En “Un credo para mis relaciones con los demás”, Gordon afirma que al respetar las necesidades de ambos participantes en la interacción, se puede crear y mantener una relación saludable en la que ambos pueden esforzarse en llegar a ser todo lo que son capaces de ser (Adams, 2006). Así que, para tener éxito en la interacción, es importante ser abierto y sincero en la comunicación.

Mediante el uso de las habilidades de interacción social los docentes pueden usar el efecto de la interacción recíproca para apoyar el aprendizaje de los alumnos, la auto-eficacia y la autonomía. Se entiende que la competencia social y emocional de un docente apoya la relación docente - alumno al igual que las relaciones fuera del aula, mantiene la gestión del aula, ayuda a los docentes a implementar el plan de estudios social y emocional, y tiene una influencia positiva sobre su propio bienestar (Jennings y Greenberg, 2009). Estos factores conducen a un mejor desempeño escolar y al bienestar psicológico de los alumnos (Durlak, Weissberg, Dymnicki, Taylor, y Schellinger, 2011).

Hay mucha investigación sobre cómo se pueden facilitar las habilidades de los niños para regular las emociones, las habilidades de cognición social, y los comportamientos 
comunicativos positivos (Durlak y Wells, 1997; Catalano, Berglund, Ryan, Lonczak, y Hawkins 2004; Greenberg et al., 2003; Wells, Barlow, y Stewart-Brown, 2003; Zins, Weissberg, Wang, y Walberg, 2004; Brock, Nishida, Chiong, Grimm, y Rimm-Kaufman, 2008; Rimm-Kaufman, Fan, Chiu, y You, 2007). Se supone que la capacidad social de un niño es producto de múltiples influencias del entorno familiar y escolar (Brophy-Herb, Lee, Nievar, y Stollak, 2007). Además, se sabe que los alumnos que tienen actitudes prosociales junto con habilidades sociales y emocionales, consiguen mejores puntuaciones académicas que sus compañeros (Durlak et al., 2011; Jiménez-Morales y López-Zafra, 2013).

Es sorprendente la poca investigación educativa que se ha publicado a nivel internacional sobre cómo los docentes pueden estudiar, desarrollar y mejorar la interacción social aún cuando se enfatizan estas habilidades en la psicología moderna del aprendizaje como herramientas claves en una comunidad de aprendizaje. Además, hay muy pocos estudios que describen los intentos por entrenar docentes a apoyar la autonomía en sus alumnos, si bien es cierto que existen algunos ejemplos (Leroy et al., 2007; Tessier, Sarrazin, y Ntoumanis, 2008).

En este estudio exploramos el desarrollo de las habilidades de interacción social en los docentes usando Maestros Eficaz y Técnicamente preparados (M.E.T.) (Gordon Training International, 2012) como una intervención con dos grupos de docentes. También queríamos abordar el modelo de Gordon desde la perspectiva de la psicología educativa moderna.

\section{Aprendizaje social y emocional (A.S.E.)}

El aprendizaje social y emocional (A.S.E.) consiste en la conciencia de sí mismo, la autogestión, la conciencia social, la gestión de relaciones y la toma de decisiones responsables (Elias et al., 1997; Zins et al., 2004; Colaboración para el aprendizaje académico, social, y emocional [CASEL], 2012). Estos componentes también se definen como factores centrales para la competencia social y emocional de los docentes (Jennings y Greenberg, 2009). Las habilidades de interacción social usadas y enseñadas en la intervención M.E.T. cubren los componentes centrales del concepto del A.S.E. (Lintunen y Gould, por publicarse).

Algunas intervenciones sobre el A.S.E. para docentes, como el estudio hecho por Leroy et al. (2007), se basan en la teoría de la autodeterminación (TAD) (Deci y Ryan, 2002). Pérez-Escoda, Filella, Alegre, y Bisquerra (2012) informaron resultados positivos del 
programa de formación con la mejora del bienestar social de los docentes y sus alumnos a través del desarrollo de su competencia emocional. En una intervención realizada por Tessier et al. (2008) los participantes aprendieron a emplear más apoyo a la autonomía y conducta neutral que los del grupo de comparación. Sin embargo, no se vieron diferencias en relación con el uso de la conducta controladora. Parecía ser muy difícil que los docentes abandonaran su conducta controladora. Sin embargo, no todo el control es dañino si se centra en la conducta dentro del aula y no en controlar el aprendizaje. Brekelmans, Mainhard, den Brok y Wubbels (2011) encontraron que los alumnos que percibían control y, simultáneamente, afiliación por parte del docente, exhibían mayores logros, compromiso más fuerte y actitudes más positivas en relación con la materia, que los alumnos que percibían en sus docentes menos de estos. En general, los docentes que son amigables y entienden a sus alumnos, pero también juegan un rol activo como líderes de la clase, son considerados como competentes en el área interpersonal. Tal control se centra en la enseñanza mientras que el controlar al alumno supone a menudo un bajo nivel de afiliación que se centra meramente en controlar, corregir y castigar.

\section{Maestros Eficaz y Técnicamente preparados (M.E.T.)}

En M.E.T., la conciencia social del docente, junto con la empatía y la comprensión, se vuelven tangibles y reales en una interacción con un alumno cuando el docente usa sus habilidades para escuchar. La escucha activa es una habilidad especial para escuchar en la cual el docente devuelve al alumno su comprensión sobre lo que el alumno ha dicho. Esto le permite al docente confirmar que él o ella entendió el mensaje, y le da la oportunidad al alumno de corregir al docente de ser necesario (Ivey, Bradford Ivey, y Zalaquett, 2009). Además, las habilidades al escuchar ayudan a los docentes a promover relaciones respetuosas y amigables con sus alumnos y realzan la toma de decisiones responsable en la que todos se sienten incluidos.

Aún más, la relación del docente con el alumno, su conciencia de sí mismo y autogestión se vuelven evidentes según él o ella usa mensajes del yo positivos o mensajes del yo de confrontación que son otras herramientas que se enseñan en el M.E.T. Los mensajes del yo positivos y de confrontación tienen tres componentes similares: una descripción del acto del alumno, el sentimiento del docente causado por este acto, y el efecto tangible de ese acto sobre el docente (Gordon, 2003, págs. 142-146). Por ejemplo, para ser capaz de construir un 
mensaje que incluya los tres componentes, el docente necesita reconocer sus propios sentimientos, necesidades, creencias y pensamientos.

Mensajes tales como ser crítico o usar la burla, algo que evitan los docentes social y emocionalmente competentes, se llaman bloqueos. Estos dañan la interacción fructífera y pueden ser mensajes polémicos ineficaces en los que el docente tiene un problema, o ser mensajes de asesoramiento ineficaces en los que el alumno tiene un problema (Gordon, 2003, pág. 136).

Además, M.E.T. incluye la idea de respaldar la autonomía y la responsabilidad propia al reducir las maneras en las que los docentes ejercen control sobre otros y las reemplaza, por ejemplo, con el método de resolución de conflictos nadie pierde y la escucha activa. Estas habilidades tienen como objetivo ayudar a los alumnos a encontrar soluciones a sus propios problemas en lugar de los docentes apropiarse de los problemas y sentimientos de los alumnos. Las habilidades del M.E.T. para respaldar la autonomía promueven la toma de decisiones responsable y la habilidad para relacionarse. Según Gordon, la escucha activa promueve la resolución de conflictos internos de los alumnos, la cual aumenta su independencia y su responsabilidad propia (Gordon, 2003, pág. 111).

La metodología del curso de M.E.T. se describe en la guía para el instructor del M.E.T. como un equilibrio entre conferencias del docente, discusiones en grupo, la puesta en común y las actividades para el desarrollo de habilidades (Adams et al., 2006, pág. 5). Por consiguiente, se enfatiza el enfoque centrado en el aprendiz. El programa del curso también incluye la discusión en grupos, deberes y ejercicios de recuperación de memoria. Ya que la reflexión puede verse como una actividad en la que se utilizan las percepciones e interpretaciones de los participantes para darle significado y mayor sentido al mundo que les rodea (Stroobants, Chambers, y Clarke, 2007), la idea de estos ejercicios es, obviamente, de guiar a los participantes a reflexionar en su propia vida y a despertar la conciencia y la comprensión sobre sí mismos.

A menudo se da por sentado que los docentes ya tienen la competencia social y emocional necesaria, junto con las habilidades para crear un entorno de apoyo, modelar la regulación de emociones, ser receptivos emocionalmente a los alumnos, formar relaciones de colaboración con los alumnos, los padres y los compañeros, manejar los problemas de conducta y resolver conflictos (Jennings y Greenberg, 2009). Elliot, Stemler, Sternberg, 
Grigorenko, y Hoffman (2011) encontraron que debido a su conocimiento tácito acumulado e inconsciente, los docentes experimentados eran ciertamente mejores que los docentes principiantes en la identificación de malas soluciones. Leroy et al. (2007) mostró también que era más probable que los docentes con experiencia dieran apoyo a la autonomía de sus alumnos y menos probable que controlaran o etiquetaran a sus alumnos. Si bien es cierto que existen algunos estudios (a saber, Pérez-Escoda et al., 2012; Talvio, Lonka, Komulainen, Kuusela, y Lintunen, 2013) que sugieren que las competencias emocionales pueden enseñarse y aprenderse, parece ser que, normalmente, la competencia social de los docentes es considerada un atributo que o existe o no existe, y podría desarrollarse en el transcurso de la vida laboral de uno, pero no mediante el estudio de la habilidad necesaria de forma sistemática.

\section{Medir el aprendizaje social y emocional durante el M.E.T.}

El análisis de videocasetes y la observación son formas típicas de medir las habilidades de comunicación y la interacción dentro del aula (Rubie-Davies, 2007). Estos métodos son importantes, sin embargo, no son siempre posibles cuando el grupo objetivo es grande. Además, es difícil capturar el momento preciso cuando se produce la conducta que nos interesa. Aún más, existe una gran variedad de situaciones de interacción retadoras en la práctica de la enseñanza cotidiana. La práctica profesional de los docentes se extiende mucho más allá del aula. Las habilidades de comunicación son necesarias en los encuentros con los padres, compañeros la administración escolar y la sociedad.

El objetivo del M.E.T. no es solo de impartir las habilidades técnicas e individuales que se usan en la interacción sino de inculcar una actitud global hacia la enseñanza. Por lo tanto, era necesaria una calificación global de la clasificación total para evaluar las habilidades de interacción social. En medicina, se ha descubierto que las listas de objetivos para medir las habilidades técnicas por separado pueden premiar la minuciosidad, pero puede que no permitan el reconocimiento de enfoques alternativos. Por otra parte, las listas de verificación no capturan el aumento de los niveles de experiencia; si se ofrece información clara sobre criterios exactos de calificación global, los investigadores podrán sacar conclusiones más válidas de lo que es posible con las listas de verificación (Regehr, MacRae, Reznick, y Szalay, 1998; Hodges, Regehr, McNaughton, Tiberius, y Hanson, 1999; Hodges y McIlroy, 2003). 
Generalmente, los comentarios de los participantes son la única fuente usada para evaluar los resultados del entrenamiento, debido a la complejidad para evaluar el entrenamiento mencionada anteriormente. En este estudio, se examinaron los efectos de la intervención de M.E.T. en docentes usando el modelo Kirkpatrick y Kirkpatrick (2006), que sugería que es importante mirar varios aspectos de los resultados de la intervención, tales como las reacciones de los participantes, el conocimiento, la aplicación del conocimiento (habilidades) y el bienestar general.

La enseñanza de las habilidades sociales y emocionales en la formación de docentes, no ha sido sistemática casi nunca, ni tampoco los docentes solían disponer de formación continua periódico. Además, son escasos los estudios sobre la enseñanza y el aprendizaje de habilidades sociales y emocionales (Lintunen, 2006). Nuestro objetivo era el de explorar la interacción social en el tipo de situaciones retadoras con las que se encuentran los docentes no solo dentro del aula, sino también con sus compañeros, con los administradores escolares y con los padres.

En este estudio estudiamos en primer lugar las reacciones de los docentes a M.E.T. En segundo lugar, se estudiaron los posibles cambios en el conocimiento de los participantes y la aplicación del conocimiento (habilidades) en el A.S.E. durante la intervención. En tercer lugar, se investigaron los posibles cambios en la experiencia de los participantes sobre sus relaciones sociales y en su bienestar durante el M.E.T.

\section{Contexto del presente estudio}

Nuestro estudio se llevó a cabo en Finlandia, que es un contexto especialmente interesante por varias razones: Los alumnos finlandeses han sido reconocidos en varias comparaciones internacionales sobre logros académicos (es decir, OECD, 2010; Mullis, Martin, Foy, y Arora, 2012). Sin embargo, se informa que las relaciones de los alumnos y el disfrute escolar son considerablemente más bajos que en otros países. También se ha mostrado en varias comparaciones internacionales sobre logros académicos que la conducta de toma de riesgos es más alta (Samdal, Dur, y Freeman, 2004; Mullis, Martin, Foy, y Arora, 2012); Mullis, Martin, Foy, y Drucker, 2012). A pesar de que se requiere que todos los docentes en Finlandia tengan un máster, los programas de formación de docentes no incluyen mucho A.S.E. Sin embargo, este tipo de cursos, a menudo, forma parte de la formación 
continua. Este estudio tiene como objetivo profundizar en nuestra comprensión del desarrollo del A.S.E. de los docentes y así ayudarles a fomentar el bienestar de los alumnos.

\section{Método}

\section{Procedimientos y participantes}

Se examinaron en este estudio los efectos de la intervención de M.E.T. en los docentes. Se organizó el M.E.T. como formación interna en las dos escuelas. El curso de formación de cuatro días se organizó en dos partes, cada parte con una duración de dos días. En ambas escuelas se llevó a cabo la primera parte de la formación durante el trimestre de otoño, a principios del año escolar, y la segunda parte durante el trimestre de primavera. Se recogieron los datos antes y después del M.E.T. dentro de un período de seis meses, tanto para los grupos de intervención como para el grupo de comparación.

Los docentes que participaron en el M.E.T. eran tutores (M.E.T. $1, n=20$ ) de educación primaria y profesores de materia (M.E.T. 2, $n=23$ ) de educación secundaria en Finlandia. El tercer grupo estaba compuesto por profesores de materia $(n=26)$ de un instituto de secundaria que no participó en el M.E.T. Sin embargo, estaban en la lista de espera para M.E.T. y estaban dispuestos a participar en el curso de M.E.T.

Al usar la prueba chi-cuadrado, no se encontraron diferencias significativas entre los grupos en términos de la experiencia laboral, la permanencia en el trabajo y los años trabajados en el mismo centro. Por consiguiente, los grupos de intervención y de comparación fueron bastante similares. Aún más, de acuerdo con las estadísticas relativas al tamaño del centro en Finlandia (Kumpulainen, 2009), los centros participantes en este estudio eran representativos de los institutos de secundaria semi-rurales finlandeses.

\section{Medidas}

Con el uso de un Cuestionario de retroalimentación del curso, se midieron las reacciones al curso M.E.T., por ejemplo, las evaluaciones sobre la aplicabilidad del curso o sobre cómo se manejó. Incluía diez ítems con una escala Likert de cinco puntos con opciones de respuesta que iban desde totalmente en desacuerdo hasta totalmente de acuerdo. "El curso cumplió mis expectativas" y "Puedo aplicar esta habilidad estudiada en el trabajo" fueron 
enunciados típicos en cuanto al contenido y objetivo del curso. Se recopilaron las opiniones sobre la gestión del curso con el uso de los siguientes enunciados "Estaba claro el procedimiento del curso" y "El formador estaba cualificado profesionalmente". Además, se le pidió a los participantes calificar sus propios niveles de energía y el curso en su totalidad. Finalmente, se calcularon los valores medios y los valores de la desviación típica de la suma de variables en las respuestas.

En la prueba de conocimientos, se les pidió a los participantes definir en sus propias palabras los conceptos centrales de las habilidades de interacción estudiados en el curso de M.E.T. Habían ocho preguntas en total: por ejemplo, se le pidió a los participantes definir qué es la escucha activa, hacer un listado de los componentes de un mensaje del yo positivo y explicar lo que implica el llamado método de nadie pierde para la resolución de conflictos, una técnica especial para la toma de decisiones responsables (Gordon, 2003). La prueba de conocimientos midió si los participantes habían aprendido la terminología de A.S.E. enseñada en el curso M.E.T. Las respuestas a la prueba de conocimientos se cuantificaron a partir de cada pregunta dando puntuaciones de 0 a 2 ó 3 puntos. Con el uso de la suma de variables en las respuestas, se calcularon los valores medios y los valores de la desviación típica.

Se desarrolló el Dealing with Challenging Interaction, DCI (Afrontamiento de las interacciones desafiantes), un método de evaluación basado en casos, para evaluar las habilidades requeridas, o la capacidad, para aplicar el conocimiento estudiado en el curso de M.E.T. Se desarrollaron siete situaciones de interacción típicas de las escuelas de los docentes las cuales se usaron para generar el cuestionario del DCI. Cada tarea consistía en la descripción de un evento común en el centro y una pregunta en la que se le pedía al encuestado describir en unas pocas líneas sus reacciones a ese evento. Por ejemplo, en un evento que envuelve confrontar la mala conducta de un alumno, se le pedía al docente describir qué haría o diría él / ella a un alumno que envía mensajes de texto durante la clase, algo que va en contra del reglamento del centro en cuestión. Otros eventos eran Dar gracias a la clase, Llevarse bien con los padres de los alumnos, Resolver un problema entre dos alumnos, Escuchar a un alumno que está preocupado, Confrontar la mala conducta de un compañero y Fijar límites. Se analizó el contenido, se cuantificaron y clasificaron las descripciones dadas por los participantes en el DCI (Weber, 1990; Frey, Botan, Friedman, y Kreps, 1992). La escucha, los mensajes del yo positivos, los mensajes del yo de confrontación, los mensajes que respaldan la autonomía y la clasificación total, representaban 
las categorías de las formas deseadas de interacción. Los bloqueos fueron la única categoría que representaba mensajes no deseados de interacción. En las categorías de los mensajes del yo de confrontación, mensajes del yo positivos y la clasificación total, el valor numérico podía ser 0, 1 ó 2 de acuerdo con la calidad de la unidad o respuesta. En otras categorías el valor numérico posible de la unidad era 0 ó 1 mostrando la existencia de esta categoría en la unidad. Finalmente, se calcularon los valores medios y los valores de la desviación típica de la suma de variables en las respuestas. En otra parte se describe más sobre el desarrollo del método de DCI (Talvio, Lonka, Komulainen, Kuusela, y Lintunen, 2012).

Para investigar la fiabilidad de la medida entre evaluadores, otro investigador analizó la mitad del material de forma paralela después de lo cual se calculó en cada categoría la probabilidad de proporción ajustada de concordancia entre dos evaluadores, el kappa de Cohen (Krippendorff, 2004). Variaba entre ,57-,86 demostrando así una fiabilidad de la medida entre evaluadores de moderada a buena.

Se midió el bienestar general modificando el perfil del bienestar escolar (Konu, 2005) que tiene como objetivo producir información sobre el bienestar en el centro. Los participantes evaluaron su bienestar con una escala de Likert que iba del 1 al 5. En nuestro cuestionario, hay cuatro categorías de preguntas: Las relaciones sociales, las relaciones con los padres, la satisfacción propia y el bienestar. La categoría de las relaciones sociales consistía en preguntas sobre las relaciones de los docentes con sus alumnos y compañeros. Las preguntas sobre las relaciones con los padres valoraba la calidad de esas relaciones. La categoría de satisfacción propia consistía en preguntas que valoraban las oportunidades que tienen los docentes para trabajar de acuerdo con sus propias aptitudes. Con las preguntas concernientes al bienestar se evaluó la falta de síntomas psicosomáticos para reflejar problemas de salud mental (ver Konu, 2005; Konu y Rimpelä, 2002; Konu, Lintonen, y Autio, 2002). Se calcularon en todas las categorías sobre bienestar los valores medios y los valores de la desviación típica de la suma de variables en las respuestas.

\section{Análisis de datos}

Se examinaron las diferencias estadísticas en los resultados entre los grupos después de la prueba y las diferencias estadísticas entre los resultados de la prueba antes de M.E.T. y la prueba después de M.E.T. con muestras dependientes del ANOVA simple. Se calcularon 
las correlaciones Pearson para investigar las correlaciones de las escalas de medida. Se usó en los análisis la SPSS 20. La consistencia interna de las escalas de medida variaban entre ,66 y ,89 (alfa de Cronbach) mostrando consistencia interna de moderada a buena. Los resultados presentados se basan en la suma de puntuaciones de la prueba después del M.E.T.

\section{Resultados}

Reacciones de los docentes a M.E.T.

La retroalimentación promedio del curso M.E.T. en la escala Likert fue de 1-5 entre los profesores de materia $M(D T)=4,06(0,33)$ y de $M(D T)=4.20(0.42)$ entre los maestros mostrando, por lo tanto, reacciones positivas al curso. Si bien es cierto que los maestros dieron más retroalimentación positiva que los profesores de materia, no se encontraron diferencias significativas entre los dos grupos participantes en M.E.T.

Cambios (e interrelaciones) de conocimientos y habilidades de A.S.E. durante el curso

El nivel de conocimiento de A.S.E. era similar en cada grupo antes de M.E.T. y no se encontraron diferencias significativas entre los grupos (Figura 1). Después de M.E.T., las puntuaciones fueron significativamente diferentes $(F(2,69)=33,98, p<, 001)$ entre los grupos, y los dos grupos de intervención puntuaron significativamente mejor que el grupo de comparación. Aún más, la prueba post-hoc mostró que había una diferencia significativa entre el grupo de comparación y los grupos de intervención, pero no entre los grupos de intervención.

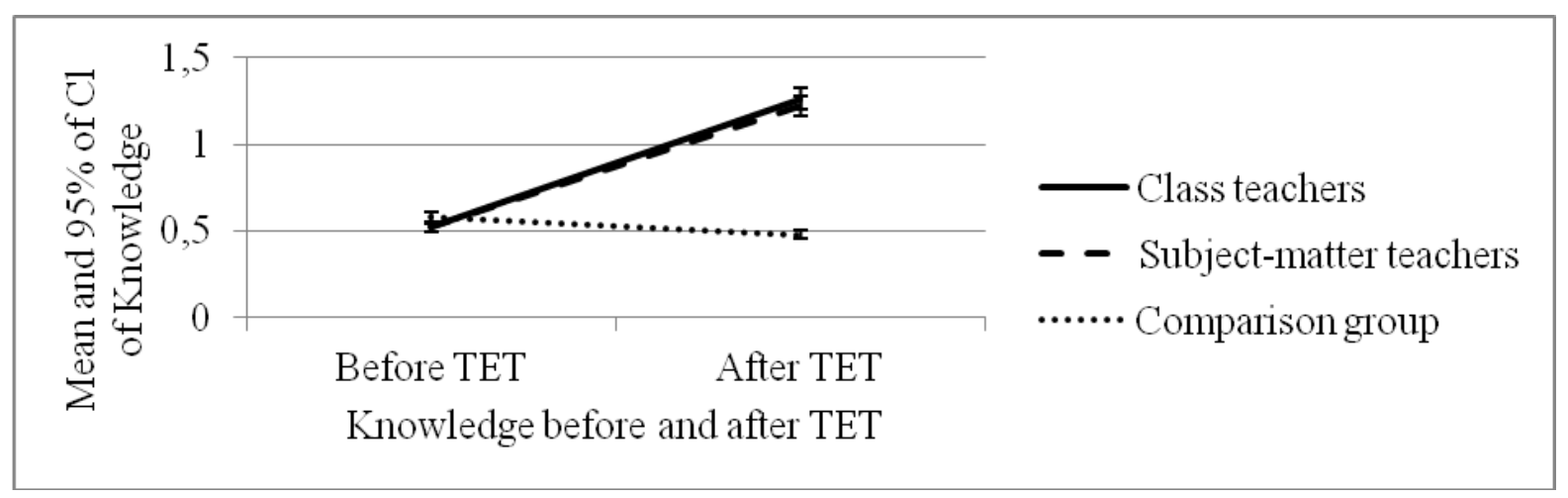

Mean and 95\% of CI of Knowledge: Media y 95\% de IC de conocimiento Before TET: Antes de MET After TET: Después de MET Knowledge: conocimiento

Class teachers: Maestros Subject-matter teachers: Profesores de secundaria Comparison group: Grupo de Comparación

Figura 1. Cambio en Conocimientos entre los grupos durante M.E.T. 
La aplicación de conocimientos (habilidades) era similar en cada grupo antes de M.E.T. y no se encontraron diferencias significativas en las puntuaciones de antes de M.E.T. entre los grupos (Figura 2). Las puntuaciones de la prueba después de M.E.T. entre los grupos discreparon significativamente las unas de las otras $(F(2,68)=67,93, p<, 001)$, con las puntuaciones más bajas entre los participantes del grupo de comparación y las más altas entre los maestros. Todas las comparaciones por parejas de las pruebas post-hoc fueron también altamente significativas. La Tabla 1 muestra con mayor detalle los cambios en las variables del DCI. En general, entre los docentes participantes de M.E.T. aumentó la variedad de maneras de interacción deseadas.

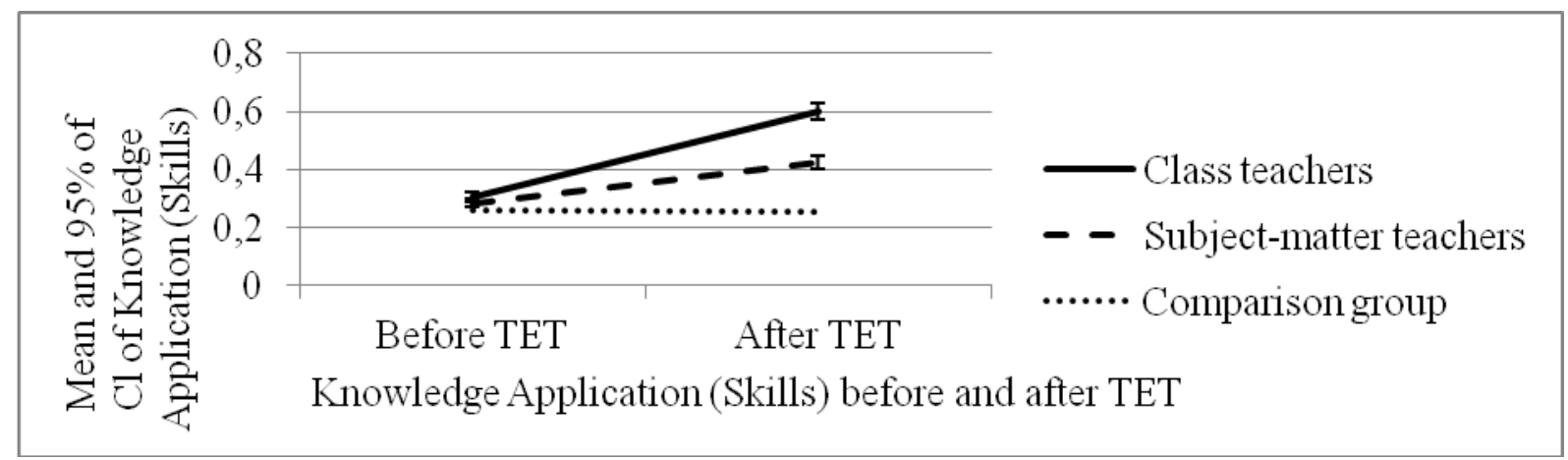

Clave: Mean and 95\% of CI of Knowledge application (skills): Media y 95\% de IC de aplicación del conocimiento (habilidades). Before TET: Antes de MET. After TET: Después de MET. Class teachers: Maestros. Subject-matter teachers: Profesores de secundaria. Comparison group: Grupo de Comparación

Figura 2. Cambio en la aplicación de Conocimientos (habilidades) entre los grupos durante M.E.T.

Se encontró una correlación significativa y moderadamente alta entre la prueba de conocimientos y la aplicación de conocimientos (habilidades) $(r=.67, p<.01)$. Por lo tanto, los docentes que sabían bien la teoría, tuvieron también éxito en aplicarla a determinadas situaciones. Además, las relaciones con los padres se correlacionaba moderadamente con la satisfacción propia $(r=, 46, p<, 001)$ y con la retroalimentación del curso $(r=, 47, p<, 001)$. En consecuencia, los docentes que sentían que sus relaciones con los padres eran buenas, experimentaron una mayor satisfacción propia en el trabajo y dieron retroalimentación más positiva sobre el curso M.E.T. Después de la intervención también se encontraron pequeñas correlaciones entre las relaciones sociales, la satisfacción propia $(r=, 32, p<, 05)$ y el bienestar $(r=, 33, p<, 01)$. No se percibieron otras asociaciones significativas después de M.E.T. 
Cambios en la experiencia de los participantes sobre el bienestar

Se descubrió un cambio significativo negativo entre las medidas de antes de M.E.T. y después de M.E.T. en el bienestar general entre los profesores de materia, en las respuestas concernientes a las relaciones con los padres $(t(21)=-3,49, p<, 01)$, y un cambio positivo significativo entre los maestros en las respuestas concernientes a la satisfacción propia $(t(15)=2,22, p<, 05)$. No se percibieron otros cambios significativos.

\section{Table 1. Resumen de las medias, desviaciones estándar, significación y tamaños de efecto de las Categorías de DNI durante el MET.}

Table 1.

Summary of Means, Standard Deviations, Significances, and Effect Sizes of the DCI Categories during TET

\begin{tabular}{|c|c|c|c|c|c|c|c|c|c|c|c|c|}
\hline \multirow[b]{4}{*}{$\begin{array}{l}\text { DCI } \\
\text { cat. }\end{array}$} & \multicolumn{4}{|c|}{ Comparison group } & \multicolumn{8}{|c|}{ Intervention group } \\
\hline & \multirow{2}{*}{\multicolumn{2}{|c|}{$\mathrm{M}(S D)$}} & \multirow[b]{3}{*}{$\mathrm{t}(25)$} & \multirow[b]{3}{*}{$d^{1}$} & \multicolumn{4}{|c|}{ TET 1} & \multicolumn{4}{|c|}{ TET 2} \\
\hline & & & & & \multicolumn{2}{|c|}{$\mathrm{M}(S D)$} & \multirow[b]{2}{*}{$\mathrm{t}(19)$} & \multirow[b]{2}{*}{$d^{1}$} & \multicolumn{2}{|c|}{$\mathrm{M}(S D)$} & \multirow[b]{2}{*}{$\mathrm{t}(22)$} & \multirow[b]{2}{*}{$d^{1}$} \\
\hline & pre & post & & & pre & post & & & pre & post & & \\
\hline LIS & $0.02(0.05)$ & $0.01(0.03)$ & -1.00 & -0.24 & $0.03(0.07)$ & $0.21(0.12)$ & $5.66 * * *$ & 1.83 & $0.02(0.05)$ & $0.09(0.10)$ & $3.76 * *$ & 0.89 \\
\hline PIM & $0.07(0.08)$ & $0.07(0.08)$ & -0.33 & 0.00 & $0.20(0.14)$ & $0.23(0.13)$ & 0.78 & 0.22 & $0.09(0.10)$ & $0.18(0.12)$ & $3.07 * *$ & 0.81 \\
\hline CIM & $0.24(0.14)$ & $0.21(0.17)$ & -0.76 & -0.19 & $0.20(0.13)$ & $0.66(0.24)$ & $10.35 * * *$ & 2.38 & $0.22(0.16)$ & $0.43(0.27)$ & $3.89 * *$ & 0.95 \\
\hline MSA & $0.06(0.07)$ & $0.06(0.09)$ & 0.44 & 0.00 & $0.09(0.11)$ & $0.15(0.08)$ & $2.34^{*}$ & 0.62 & $0.06(0.09)$ & $0.13(0.09)$ & $3.19 * *$ & 0.78 \\
\hline RB & $0.60(0.20)$ & $0.57(0.19)$ & -0.57 & -0.15 & $0.49(0.22)$ & $0.22(0.16)$ & $-4.75 * * *$ & -1.40 & $0.53(0.13)$ & $0.46(0.20)$ & 1.53 & 0.42 \\
\hline GR & $0.77(0.22)$ & $0.74(0.20)$ & -0.55 & -0.14 & $0.80(0.18)$ & $1.56(0.24)$ & $13.27 * * *$ & 3.58 & $0.84(0.23)$ & $1.18(0.34)$ & $3.73 * *$ & 1.17 \\
\hline
\end{tabular}

Note : LIS = Listening, PIM = Positive I-Messages, CIM = Confrontation I-Messages, MSA = Messages supporting autonomy, RB = Road blocks, GR = Global rating. TET $1=$ Class teachers, TET $2=$ Subject-matter teachers. $d^{1}=$ Cohen's $d . * p<.05 . * * p<.01 . * * * p<.001$.

Clave: Comparison Group: Grupo de comparación. Intervention Group: Grupo de intervención. TET: MET. M(SD): M(DT). LIS: Escucha; PIM: Mensajes positivos del yo; CIM: Mensajes de confrontación del yo; MSA: Mensajes que apoyan la autonomía; RB: Bloqueos; GR: Puntuación Global; TET 1: Maestros; TET 2: Profesores; d: de de Cohen. 


\section{Discusión y conclusión}

Para resumir los resultados, las reacciones de los docentes hacia el M.E.T. fueron positivas. Además, el conocimiento sobre el A.S.E. de los docentes participantes en M.E.T. aumentó significativamente. No se percibió diferencia entre las medidas antes de la prueba y después de la prueba entre el grupo de comparación.

Aún más, la aplicación de conocimiento (habilidades) mejoró entre los docentes después de su participación en M.E.T. Tanto los maestros como los profesores de materia que asistieron a M.E.T. describieron significativamente más a menudo la escucha, los mensajes del yo de confrontación y los mensajes de respaldo de la autonomía, que los docentes que no asistieron a M.E.T. Además, de los docentes que participaron en M.E.T. los mensajes del yo positivos aumentaron entre los profesores de materia, mientras que una forma de interacción no deseada, o los mensajes en la categoría de bloqueos, disminuyeron significativamente entre los maestros en la medida después de la prueba. En consecuencia, en ambos grupos de intervención, la puntuación de calificación global de los mensajes de los docentes aumentó después de M.E.T. En el grupo de comparación no se encontró ninguna diferencia en ninguna categoría ni en la prueba de antes de M.E.T. ni en la prueba de después de M.E.T. Este resultado es interesante, ya que durante la intervención no solo aumentó la conducta de apoyo a la autonomía, sino que se percibieron signos decrecientes de la conducta controladora (disminución de bloqueos). Este resultado es diferente al de Tessier et al. (2008). En general, los resultados en términos del nivel de conocimiento aplicado coincidieron con los objetivos del curso. Los participantes que asistieron a M.E.T mostraron progreso en las categorías basadas en la teoría que fueron el enfoque del curso M.E.T. Además, las respuestas en la categoría de mensajes de respaldo a la autonomía aumentaron, si bien es cierto que no estaba incluida de manera explícita en el plan de estudios del curso. Un detalle interesante es que, aún antes del curso M.E.T., los maestros usaban más mensajes del yo positivos que los profesores de materia. Esto podría reflejar una diferencia entre las materias principales que se imparten en la formación docente. La materia principal para los alumnos de profesorado de clases es pedagogía, la cual puede abarcar una gran cantidad de habilidades didácticas, mientras que los profesores de materia tienen bastante menos formación en pedagogía. Además, es posible que esos candidatos con un enfoque pedagógico de la enseñanza busquen formación como maestro en lugar de formación para el profesorado con más orientación académica en una materia en específico. Aún más, en la escuela primaria, la comunidad de 
práctica con docentes y padres podría apoyar la formación orientada a la pedagogía. Estas diferencias también podrían explicar por qué después del curso M.E.T., no disminuyeron tanto los bloqueos entre los profesores de materia como lo hicieron entre los maestros.

Finalmente, la cuarta categoría, el bienestar general, mostró un cambio positivo en la satisfacción propia entre los maestros, pero entre los profesores de materia aumentó la falta de satisfacción con sus relaciones con los padres. Es posible que la formación hiciera que los profesores de materia estuvieran más conscientes sobre las complicaciones que tenían con los padres de sus alumnos. Es comprensible que no se percibieran ningunos otros cambios en esta categoría durante el curso M.E.T. Se organizó la última medida para inmediatamente después de la segunda parte del curso M.E.T. Aún cuando la primera parte del curso M.E.T. se realizó seis meses antes, la mitad del contenido del curso y, en particular, la parte que consistía en aplicar las habilidades, se dieron inmediatamente antes del segundo test. En consecuencia, habría sido necesario más tiempo para que los docentes utilizaran las habilidades de M.E.T. en su entorno de trabajo.

Por otro lado, queríamos verificar si el cambio en términos de conocimiento y habilidades se podía explicar con un cambio en el bienestar general durante el curso M.E.T. En este caso, aún cuando el bienestar general entre los profesores de materia era más bajo que entre los maestros, ellos pudieron aprender el conocimiento y las habilidades que se les enseñaron. Sin embargo, las mejores puntuaciones en bienestar general podrían explicar por qué los maestros tenían mayor éxito en el aprendizaje de estas habilidades.

\section{Reflexiones metodológicas}

Las correlaciones entre los resultados de la prueba de conocimientos y los resultados del método DCI, al igual que las correlaciones entre las escalas de bienestar general, mostraron buen criterio orientado a la validez de las medidas. También encontramos apoyo en estudios previos en cuanto a que las intervenciones que pretenden mejorar las habilidades de interacción pueden ser exitosas (Barton-Arwood, Morrow, Lane, y Jolivette, 2005; Tessier et al., 2008, Pérez-Escoda et al., 2012; Talvio et al., 2013).

Si bien es cierto que la retroalimentación positiva del curso se correlacionó con una experiencia positiva en términos de las relaciones con los padres de los alumnos, los resultados de los datos de retroalimentación del curso no demostraron éxito en el de las 
habilidades estudiadas en M.E.T. Sin embargo, es comprensible, ya que solo se recogieron datos de retroalimentación sobre el curso de los docentes con formación M.E.T., y no había mucha variedad en los datos debido a que la mayoría de los participantes estaban bastante satisfechos con el curso. Además, en la formación sobre interacción, es probable que la gente tenga que reconocer sus limitaciones $\mathrm{y}$, puede que esto, a la vez, les haga sentir algo incómodos. Esto resulta en que, aún cuando las reacciones sean menos positivas, se puedan alcanzar los objetivos de aprendizaje.

El lector debe tener presente que solo se estudió un pequeño grupo de docentes. Se necesita hacer más investigación en diversos centros y países antes de poder generalizar sobre estos resultados. Además la composición de este estudio, hasta cierto punto, se pareció al diseño cuasi-experimental con el grupo de comparación (Shadish, Cook, y Campbell, 2002), pero como el grupo de comparación consistió solamente de profesores de materia y la muestra no fue aleatoria, no se correspondió en su totalidad con el grupo de intervención que consistía de maestros y profesores de materia. Sin embargo, el diseño cuasi-experimental adaptado nos dio alguna información interesante sobre cómo la formación básica del docente puede afectar el resultado de la formación en interacción. Nuestro objetivo no era solo de explorar las diferencias entre los grupos de intervención y el grupo de comparación, sino de también investigar las diferencias entre los tres grupos participantes en el estudio. Por lo tanto, podría ser interesante investigar las diferencias potenciales entre los docentes de primaria y los de secundaria, participantes en M.E.T., teniendo grupos de comparación para ambos grupos de intervención.

Otra limitación fue que esta investigación no proveyó ninguna información sobre las acciones de los docentes en las aulas, únicamente sus propias descripciones sobre sus acciones en situaciones hipotéticas. Por otro lado, al valorar el rendimiento de estudiantes de medicina durante la formación clínica, parecía que era más viable, fiable y válido recopilar material a través de muestreo durante encuentros habituales en el día laboral que los datos de una simple escala de calificación global completada a intervalos poco frecuentes a través de un supervisor (Turnbull, MacFadyen, van Barneveld, y Norman, 2000) Además, se ha sugerido que deberían usarse para la enseñanza ejemplos de situaciones prácticas reales para ilustrar la teoría previamente estudiada (Norman, 2009).

Con el fin de observar las acciones reales de los docentes en el aula, sería necesario reunir material de investigación por medio de la observación o de grabaciones. Sin embargo, 
habría sido difícil la recolección de ese material por las siguientes razones: Primero, existe un problema de base de referencia. Las habilidades que son el enfoque de M.E.T. se utilizan mayormente cuando un docente o un alumno afronta un problema. Este tipo de situaciones puede no ocurrir con mucha frecuencia. Segundo, estas habilidades no se pueden poner en práctica inmediatamente después del curso. Tercero, para poder grabar cada participante en este estudio habríamos necesitado muchos más recursos. Ya que el propósito de este estudio era de centrarnos en el cambio de un grupo participante en el curso, el grupo de preguntas usado se veía como una forma práctica para recoger material. Por consiguiente, estos resultados podrían indicar un cambio en el pensamiento y la comprensión de los docentes debido a la intervención, el curso M.E.T. Sin embargo, la investigación cualitativa basada en material de video sobre algunos participantes proporcionaría información muy interesante acerca de cómo los docentes participantes en M.E.T. han aplicado las habilidades conseguidas en sus interacciones en la vida real con los alumnos, los padres y otros compañeros.

\section{Reflexiones teóricas}

De acuerdo con el presente estudio, el curso M.E.T. parece ser una forma efectiva para los docentes mejorar su conocimiento sobre A.S.E. y su capacidad para aplicarlo. La teoría y las habilidades provistas por M.E.T. a veces son consideradas como solo un conjunto de técnicas de gestión del aula. Sin embargo, de acuerdo con nuestros hallazgos, los efectos podrían ser más amplios. Se comenzó a usar M.E.T. en los años 1970s. Como representante de la individualista psicología humanista, Thomas Gordon (alumno y compañero de Carl Rogers) se centró en los recursos potenciales del individuo. Nosotros sugerimos que el apoyo de la autonomía es un acto de colaboración. Al permitir la autonomía de los alumnos, mostrar respeto y dejar la responsabilidad del aprendizaje sobre los alumnos, los docentes les dan la oportunidad de desarrollar su agencia. En consecuencia, el objetivo general de M.E.T. es de aumentar las formas constructivas y participativas de comunicación, la toma de decisiones y el apoyo a la autonomía tanto de los alumnos como de los docentes.

\section{Agradecimientos}

Esta investigación fue financiada por una beca para el primer autor por parte de la Fundación Cultural Finlandesa, y parcialmente financiada por el proyecto RYM Indoor Environment 462054 de la Agencia finlandesa de financiación para la tecnología y la innovación, www.indoorenvironment.org. 


\section{Referencias}

Adams, L., Denslow, K. K., Emmons, S., Tyrrell, P., Miller, K., y Burch, N. (2006). Dr. Thomas Gordon's teacher effectiveness training (T.E.T.). Instructor guide. Solana Beach, CA: Gordon Training International.

Adams, L. (2006). Dr. Thomas Gordon's teacher effectiveness training (T.E.T.). Workbook. Solana Beach, CA: Gordon Training International.

Aspinwall, L.G. y Staudinger, U.M., (eds). (2003). A Psychology of Human Strengths: Fundamental Questions and Future Directions for a Positive Psychology.Washington DC: American Psychological Association.

Bandura, A. (2006). Toward a psychology of human agency. Perspectives on Psychological Science, 1(2), 164180.doi: 10.1111/j.1745-6916.2006.00011.x

Barton-Arwood, S., Morrow, L., Lane, K., y Jolivette, K. (2005).Project IMPROVE: Improving teachers' ability to address students' social needs. Education and Treatment of Children, 28(4), 430-443.

Bird, K. A., y Sultmann, W. F. (2010). Social and emotional learning: Reporting a system approach to developing relationships, nurturing well-being and invigorating learning. Educational y Child Psychology, 27(1), 143155.

Brekelmans, M., Mainhard, T., den Brok, P., y Wubbels, T. (2011). Teacher control and affiliation: Do students and teachers agree? Journal of Classroom Interaction, 46(1), 17-26.

Brock, L. L., Nishida, T. K., Chiong, C., Grimm, K. J., y Rimm-Kaufman, S. E. (2008). Children's perceptions of the classroom environment and social and academic performance: A longitudinal analysis of the contribution of the responsive classroom approach. Journal of School Psychology, 46(2), 129-149. doi: 10.1016/j.jsp.2007.02.004

Brophy-Herb, H. E., Lee, R. E., Nievar, M. A., y Stollak, G. (2007). Preschoolers' social competence: Relations to family characteristics, teacher behaviors and classroom climate. Journal of Applied Developmental Psychology, 28(2), 134-148. doi: 10.1016/j.appdev.2006.12.004

Bruner.J. (1996). The culture of education. Cambridge, MA: Harward University Press.

Cantor, N. (2003). Constructive cognition, personal goals, and the social embedding of personality. En Aspinwall, L.G. y Staudinger, U.M., (Eds). A Psychology of Human Strengths: Fundamental Questions and Future Directions for a Positive Psychology, Washington DC: American Psychological Association.

Catalano, R. F., Berglund, M. L., Ryan, J. A. M., Lonczak, H. S., y Hawkins, J. D. (2004). Positive youth development in the United States: Research findings on evaluations of positive youth development programs. The Annals of the American Academy of Political and Social Science, 591, 98-124. doi: 10.1037/15223736.5.1.515a

Collaborative for academic, social and emotional learning (2012). Implementation » overview. Consultado en http://www.casel.org/implement/index.php

Deci, E. L. y Ryan, R. M. (2002). Overview of self-determination theory: an organismic dialectical perspective. En E.L. Deci y R.M. Ryan (Eds.), Handbook of self-determination research. Rochester NY: University of Rochester Press. 
Durlak, J. A., Weissberg, R. P., Dymnicki, A. B., Taylor, R. D., y Schellinger, K. B. (2011). The impact of enhancing students' social and emotional learning: A Meta-Analysis of School-Based universal interventions. Child Development, 82(1), 405-432. doi: 10.1111/j.1467-8624.2010.01564.x

Durlak, J. A., y Wells, A. M. (1997). Primary prevention mental health programs for children and adolescents: A meta-analytic review. American Journal of Community Psychology, 25(2), 115-152. doi: 10.1023/A:1024654026646

Edwards, A. (2005). Relational agency: Learning to be a resourceful practitioner International Journal of Educational Research, 43(3), 168-182. doi: 10.1016/j.ijer.2006.06.010

Elias, M. J., Zins, J. E., Weissberg, T. P., Frey, K. S., Greenberg, M. T., Haynes, N.M.,

Kessler, R., Schwab-Stone, M. E., y Shriver, T. P. (1997). Promoting social and emotional learning: Guidelines for educators. Alexandria, VA: Association for Supervision and Curriculum Development.

Elliott, J. G., Stemler, S. E., Sternberg, R. J., Grigorenko, E. L., y Hoffman, N. (2011). The socially skilled teacher and the development of tacit knowledge. British Educational Research Journal, 37(1), 1-21. doi: $10.1080 / 01411920903420016$

Frey, L. R., Botan, C. H., Friedman, P. G., y Kreps, G. L. (cop. 1992.). Interpreting communication research: A case study approach. Englewood Cliffs, NJ: Prentice Hall.

Gordon Training International (2012). T.E.T. teacher effectiveness program. Consultado en:

http://www.gordontraining.com/teachertrainingprogram.html

Gordon, T. (2003).Teacher effectiveness training. New York: Three rivers press.

Greenberg, M. T., Weissberg, R. P., O Brien, M. U., Zins, J. E., Fredericks, L., Resnik, H., y

Elias, M. J. (2003). Enhancing school-based prevention and youth development through coordinated social, emotional, and academic learning. American Psychologist, 58(6), 466-474. doi: 10.1037/0003-066X.58.67.466

Hodges, B., y McIlroy, J. H. (2003). Analytic global OSCE ratings are sensitive to level of training. Medical Education, 37(11), 1012-1016. doi: 10.1046/j.1365-2923.2003.01674.x.

Hodges, B., Regehr, G., McNaughton, N., Tiberius, R., y Hanson, M. (1999). OSCE checklists do not capture increasing levels of expertise. Academic Medicine, 74(10), 1129-1134. doi: 10.1097/00001888-19991000000017

Ivey, A. E., Bradford Ivey, M., y Zalaquett, C. P. (2009).Intentional interviewing and counselling: Facilitating client development in a multicultural society ( $7^{\mathrm{a}} \mathrm{ed}$.). Belmont CA: Cengage Learning.

Jennings, P. A., y Greenberg, M. T. (2009). The prosocial classroom: Teacher social and emotional competence in relation to student and classroom outcomes. Review of Educational Research, 79(1), 491-525.doi: $10.3102 / 0034654308325693$

Jiménez-Morales, M., yLópez-Zafra, E. (2013). Impact of Perceived Emotional Intelligence, Social Attitudes and Teacher Expectations on Academic Performance. Electronic Journal of Research in Educational Psychology $11(1), 75-98$. 
Kirkpatrick, D. L., y Kirkpatrick, J. D. (2006). Evaluating training programs: The four levels $\quad\left(3^{\mathrm{a}} \quad\right.$ ed.). $\quad$ San Francisco, CA: Berrett-Koehler Publishers, Inc.

Konu, A. (2005). School well-being profile. Consultado en http://www10.edu.fi/hyvinvointiprofiili/infoeng.html.

Konu, A. I., Lintonen, T. P., y Autio, V. J. (2002). Evaluation of well-being in schools - A multilevel analysis of General subjective well-being. School Effectiveness \& School Improvement, 13(2), 187-200. doi: 10.1076/sesi.13.2.187.3432

Konu, A., y Rimpelä, M. (2002). Well-being in schools: A conceptual model. Health Promotion International, 17(1), 79-87. doi:10.1093/heapro/17.1.79

Krippendorff, K. (2004). Content analysis: An introduction to its methodology (2a ed.). Thousand Oaks, CA: Sage.

Kumpulainen, T. (2009). Koulutuksenmäärällisetindikaattorit 2009. [Los indicadores cuantitativos de la educación, 2009]. Helsinki: Opetushallitus.

Leroy, N., Bressoux, P., Sarrazin, P., yTrouilloud, D. (2007). Impact of teachers' implicit theories and perceived pressures on the establishment of an autonomy supportive climate. European Journal of Psychology of Education 22(4), 529-545. doi: 10.1007/BF03173470

Lintunen, T. (2006). Social and emotional learning in the school physical education context. En F. Boen, B. De Cuypery J. Opdenacker (Eds.). Current research topics in exercise and sport psychology in Europe (11 ${ }^{\mathrm{a}}$ ed., pp. 25-33). Leuven: Lannoo Campus.

Lintunen T. y Gould, D. (en prensa). Developing social and emotional skills. En A. Papaioannou \& D. Hackfort (eds.). Fundamental Concepts in Sport and Exercise Psychology. London: Routledge.

Lonka, K., y Ahola, K. (1995). Activating instruction: How to foster study and thinking skills in higher education? European Journal of Psychology of Education, 10(4), 351-368.doi:10.1007/BF03172926

Mullis, I.V.S., Martin, M.O., Foy, P., y Arora, A. (2012). TIMSS 2011 International Results in Mathematics. Chestnut Hill, MA: TIMSS \& PIRLS International Study Center, Boston College.

Mullis, I.V.S., Martin, M.O., Foy, P., y Drucker, K.T. (2012). The PIRLS 2011 International Results in Reading. Chestnut Hill, MA: TIMSS \& PIRLS International Study Center, Boston College.

Norman, G. (2009). Teaching basic science to optimize transfer. Medical Teacher, 31(9), 807-811. doi:10.1080/01421590903049814

OECD. (2010). PISA 2009 results: What students know and can do. Organisation for Economic Co-operation and Development.

Pérez-Escoda, N., Filella, G., Bisquerra, R., y Alegre, A. (2012). Developing the emotional competence of teachers and pupils in school contexts. Electronic Journal of Research in Educational Psychology 10 (3), 1183-1208.

Pietarinen, J., Soini, T., Pyhältö, K., y Jindal-Snape, D. (2010). Learning and well-being in transitions: How to promote pupils' active learning agency? Educational Transitions. Moving Stories from Around the World, 25(2), 143-158. 
Regehr, G., MacRae, H., Reznick, R. K., ySzalay, D. (1998). Comparing the psychometricproperties of checklists and global rating scales for assessing performance on an OSCE-format examination. Academic Medicine, 73(9), 993-997. doi: 10.1097/00001888-199809000-00020

Rimm-Kaufman, S. E., Fan, X., Chiu, Y., y You, W. (2007). The contribution of the responsive classroom approach on children's academic achievement: Results from a three year longitudinal study. Journal of School Psychology, 45(4), 401-421.doi: 10.1016/j.jsp.2006.10.003

Rubie-Davies, C. (2007). Classroom interactions: Exploring the practices of high- and low-expectation teachers. British Journal of Educational Psychology, 77(2), 289-306.Doi:10.1348/000709906X101601

Ryan, R. M., yDeci, E. L. (2000). Self-determination theory and the facilitation of intrinsic motivation, social development, and well-being. American Psychologist, 55(1), 68-78.

Samdal, O., Dur, W. y Freeman, J. (2004).School. In Currie C., Roberts C., Morgan M. Smith R., Settertobulte W., Samdal O. y Rasmussen V. (Ed.), Young people's health in context: Health behaviour in school-aged children (HBSC) study: International report from the 2001/2002 survey (pp. 42-51). Copenhagen: WHO Regional Office for Europe.

Sfard, A. (1998). On two metaphors for learning and the dangers of choosing just one, Educational Researcher, 27(2), 4-13.doi: 10.3102/0013189X027002004

Shadish, W. R., Cook, T. D., y Campbell, D. T. (2002.). Experimental and quasi-experimental designs for generalized causal inference. Boston: Houghton Mifflin.

Stroobants, H., Chambers, P., y Clarke, B. (2007). Reflective journeys: A fieldbook for facilating life-long learning in vocational education and training. Leuven: Acco.

Talvio, M., Lonka, K., Komulainen, E., Kuusela, M., y Lintunen, T. (2012). The development of the Dealing with Challenging Interaction (DCI) method to evaluate teachers' social interaction skills. Procedia - Social and Behavioral Sciences 69, 621-630.doi: 10.1016/j.sbspro.2012.11.454

Talvio, M., Lonka, K., Komulainen, E., Kuusela, M., y Lintunen, T. (in press).The development of teachers' responses to challenging situations during interaction training. Teacher development.

Tessier, D., Sarrazin, P., y Ntoumanis, N. (2008). The effects of an experimental programme to support students' autonomy on the overt behaviours of physical education teachers. European Journal of Psychology of Education - EJPE 23(3), 239-253. doi: 10.1007/BF03172998

Turnbull, J., MacFadyen, J., van Barneveld, C., y Norman, G. (2000). Clinical work sampling. JGIM: Journal of General Internal Medicine, 15(8), 556-561. doi: 10.1046/j.1525-1497.2000.06099.x

Vygotsky, L. (1978). Mind in society.The development of higher psychological processes. Cambridge: Harvard University Press.

Weber, R. P. (1990). Basic content analysis (2a ed.). Beverly Hills CA: Sage.

Wells, J., Barlow, J., y Stewart-Brown, S. (2003). A systematic review of universal approaches to mental health promotion in schools. Health Education, 103(4), 197-220. doi: 10.1108/09654280310485546

Zins, J. E., Weissberg, R. P., Wang, M. C., y Walberg, H. J. (Eds.). (2004). Building academic success on social and emotional learning: What does the research say? New York: Teachers College Press. 